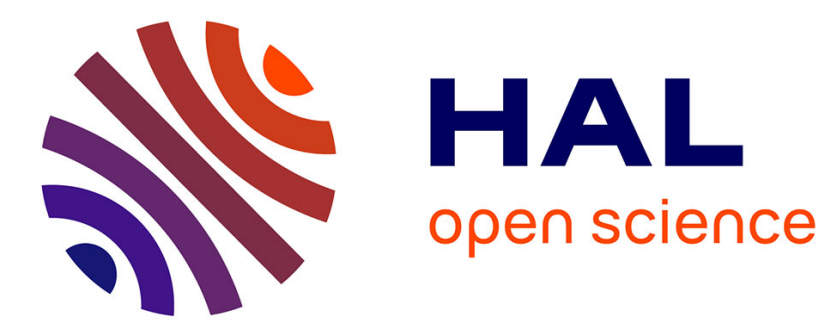

\title{
Le tramway de Tours: peut-on comparer celui de 2013 avec celui de 1913 ?
}

Jean-Marie Beauvais, Nadine Polombo

\section{To cite this version:}

Jean-Marie Beauvais, Nadine Polombo. Le tramway de Tours: peut-on comparer celui de 2013 avec celui de 1913 ?. Transports urbains: mobilité, réseaux, territoires, 2014, 124, pp.8-19. halshs01060129

\section{HAL Id: halshs-01060129 \\ https://shs.hal.science/halshs-01060129}

Submitted on 3 Sep 2014

HAL is a multi-disciplinary open access archive for the deposit and dissemination of scientific research documents, whether they are published or not. The documents may come from teaching and research institutions in France or abroad, or from public or private research centers.
L'archive ouverte pluridisciplinaire HAL, est destinée au dépôt et à la diffusion de documents scientifiques de niveau recherche, publiés ou non, émanant des établissements d'enseignement et de recherche français ou étrangers, des laboratoires publics ou privés. 


\section{Le tramway de Tours : peut-on comparer celui de 2013 avec celui de 1913 ?}

\section{Jean-Marie BEAUVAIS \\ économiste des \\ transports (Beauvais \\ Consultants) \\ Nadine Polombo \\ maitre de conférences \\ (Université de Tours) \\ Cet article original vise a comparer l'ancien et le nouveau réseau dà un siècle d'intervalle, dans de nombreux domaines: tracés des lignes, choix techniques, volume d'offre, vitesse commerciale, tarification, fréquentation et design. Permanences et ruptures peuvent etre ainsi mises en evidence.}

\section{Introduction}

Pourquoi 2013 ? C'est l'année de la mise en service de la première ligne de tramway, tout au moins du nouveau réseau car il y eut, en 1877, la mise en service de la première ligne de l'ancien réseau.

Pourquoi 1913 ? Parce que cet ancien réseau a connu son apogée en 1913, la première guerre mondiale ayant mis fin aux projets (extension vers Esvres, création d'une ligne circulaire pour les marchandises).

Il ne s'agit pas de retracer l'histoire du tramway de 1913 à 2013 mais de confronter deux moments d'histoire. Entre ces deux photos, juste un siècle soit environ trois générations.

Pourquoi une comparaison? Pour mettre en évidence les éventuelles avancées en matière de réponses techniques aux besoins de mobilité des habitants. Pour mettre en évidence les éventuelles spécificités tourangelles qui traverseraient les époques.

Comme toute comparaison, celle-ci a ses limites. En effet, d'autres moyens de transport motorisés sont apparus entre ces deux dates: l'autobus qui est arrivé à Tours en 1931 et la voiture particulière qui existait déjà (en nombre très limité, puisque le parc d'Indre-et-Loire ne comptait que 425 automobiles en 1913) mais qui a bénéficié, avec la société de consommation, d'une large diffusion pour atteindre 300.000 unités au niveau du département de nos jours.

La source la plus utilisée pour le volet historique est sans conteste le numéro spécial consacré aux tramways de Tours que la revue de la Fédération des amis des chemins de fer secondaires (F.A.C.S.) a publié en $1970^{1}$.

\section{1 - Le réseau}

En 1913, le réseau comprenait $54 \mathrm{~km}$ de lignes (voir Figure 1 « Extension du tramway et de l'urbanisation en $1913 »)$ :

\footnotetext{
${ }^{1}$ Et notamment l'article de J. Metz qui lui-même s'appuie sur un article de J.-C. Vaudois publié en 1960 dans la même revue et sur des cartes dressées par P. Laederich.
} 
- 4 lignes urbaines (les lignes A, B, C, D) totalisant $18 \mathrm{~km}$;

- 4 lignes suburbaines (la ligne de Vouvray, la ligne de Fondettes et de Luynes, la ligne d'Azay-sur-Cher et la ligne de la Tranchée) totalisant $36 \mathrm{~km}$.

Aujourd'hui, la ligne qui va de Joué-lès-Tours à Tours-Nord est longue de 15 km (voir Figure 2 «Extension du tramway et de l'urbanisation en 2013 »).

Le réseau était ainsi 3,6 fois plus étendu hier qu'aujourd'hui. Pourtant la population était moindre. En effet, en 1913, le réseau desservait 14 communes :

- Au nord de la Loire : Vouvray, Rochecorbon, Ste-Radegonde, St-Symphorien, St-Cyr-surLoire, Fondettes et Luynes ;

- Entre la Loire et le Cher : St-Pierre-des-Corps et Tours

- Au sud de la Loire : Azay-sur-Cher, Veretz, Larcay, St-Avertin et Joué-lès-Tours.

Au recensement de 1911, la population de l'ensemble de ces 14 communes atteignait 94.811 habitants. En 2011, la population de ces communes (devenues 12 à la suite du rattachement à Tours de Ste-Radegonde et de St-Symphorien en 1964) atteint 248.641 habitants. Le nombre d'habitants a donc été multiplié par 2,6 en 100 ans.

Un réseau qui était 3,6 fois plus étendu alors que la population était 2,6 fois moindre, cela veut dire que l'offre en nombre de kilomètres de ligne par habitant a été divisée par 9,5. De ce point de vue, nos anciens étaient donc en avance.

\begin{tabular}{|l|r|r|}
\hline & 1913 & \multicolumn{2}{|l|}{2013} \\
\hline Population des communes desservies par le tramway (habitants) & 94.811 & 248.641 \\
\hline Longueur des lignes de tramway (kilomètres) & 54 & 15 \\
\hline Nombre de kilomètres de ligne par centaine de milliers d'habitants & $\mathbf{5 7}$ & $\mathbf{6}$ \\
\hline
\end{tabular}

Durant le siècle dernier l'urbanisation a gagné du terrain : le bâti continu a maintenant franchi le Cher. C'est ce qui apparait clairement lorsqu'on compare la tache urbaine actuelle avec celle de 1913.

\section{Mattodologia de reprisentation}

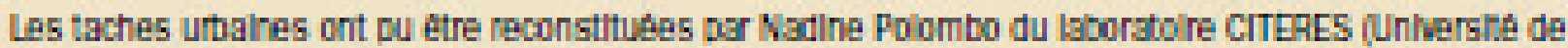

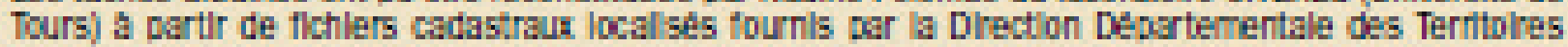

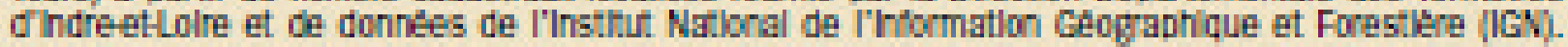
Cornalssart la date de constructuon du batl, on rele entre eux les batiments constrults avant 1913 ou apres 1913 dar des disques aprefes, la tache globe obtenue est ensulie llssed. Le resultat compurte des blals et des lnoerti.udes les a la fols a la methode et aur donnees. Le chole des tyoes de bat ments a vendre en compte, de l'entze de base: tatiment ou zarcelle, le cholx des talles des zones tampons sucoesshes, Infuencent la tome de la tache urbahe. Les donness de dates du cadaste sont Incom-

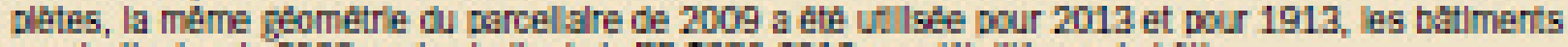
constrults depuls 2008 sont eatrat ts de la BD TOP0 2012 peu detallese sur le bat. 
Lorsqu'on projette le tracé du réseau de 1913 sur la carte de la tache urbaine de l'époque, on note que le tramway ne dessert pas que des zones denses. En direction de Vouvray comme en direction de Luynes, l'urbanisation était généralement limitée à une bande le long de la voie. En direction, d'Azay-sur-Cher, la densité était encore plus faible.

Inversement, lorsqu'on projette le tracé de la ligne de 2013 sur la carte de la tache urbaine de notre époque, on voit que certaines zones denses ne sont pas desservies. C'est le cas, de Rochecorbon, de Saint-Pierre-des-Corps, de Saint-Cyr, de La Riche, de Ballan, de St-Avertin, et de Chambray-lès-Tours. Des extensions du nouveau tramway ne pourraient-elles pas être envisagées en direction de ces communes?

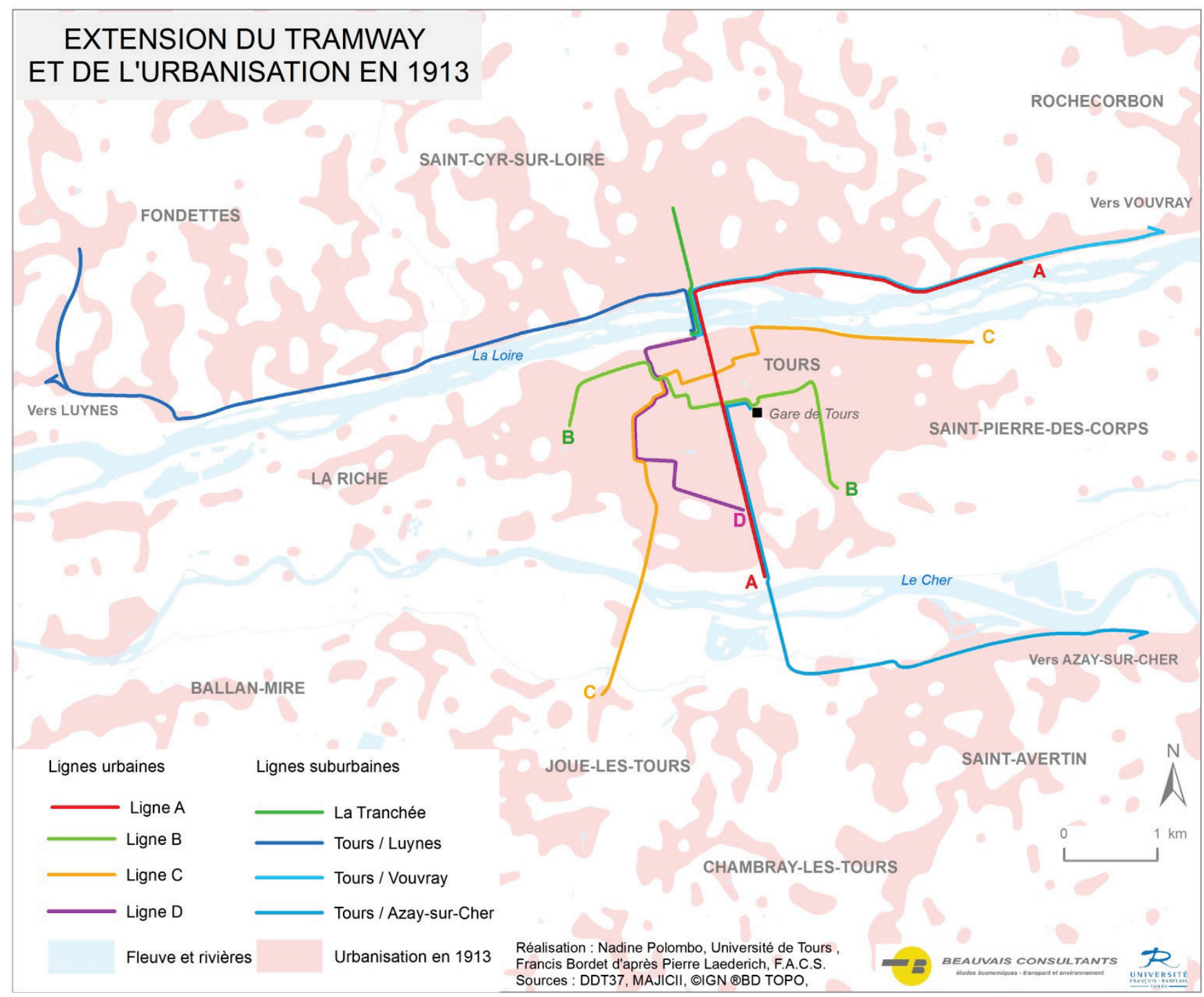

Figure 1 «Extension du tramway et de l'urbanisation en 1913 » 


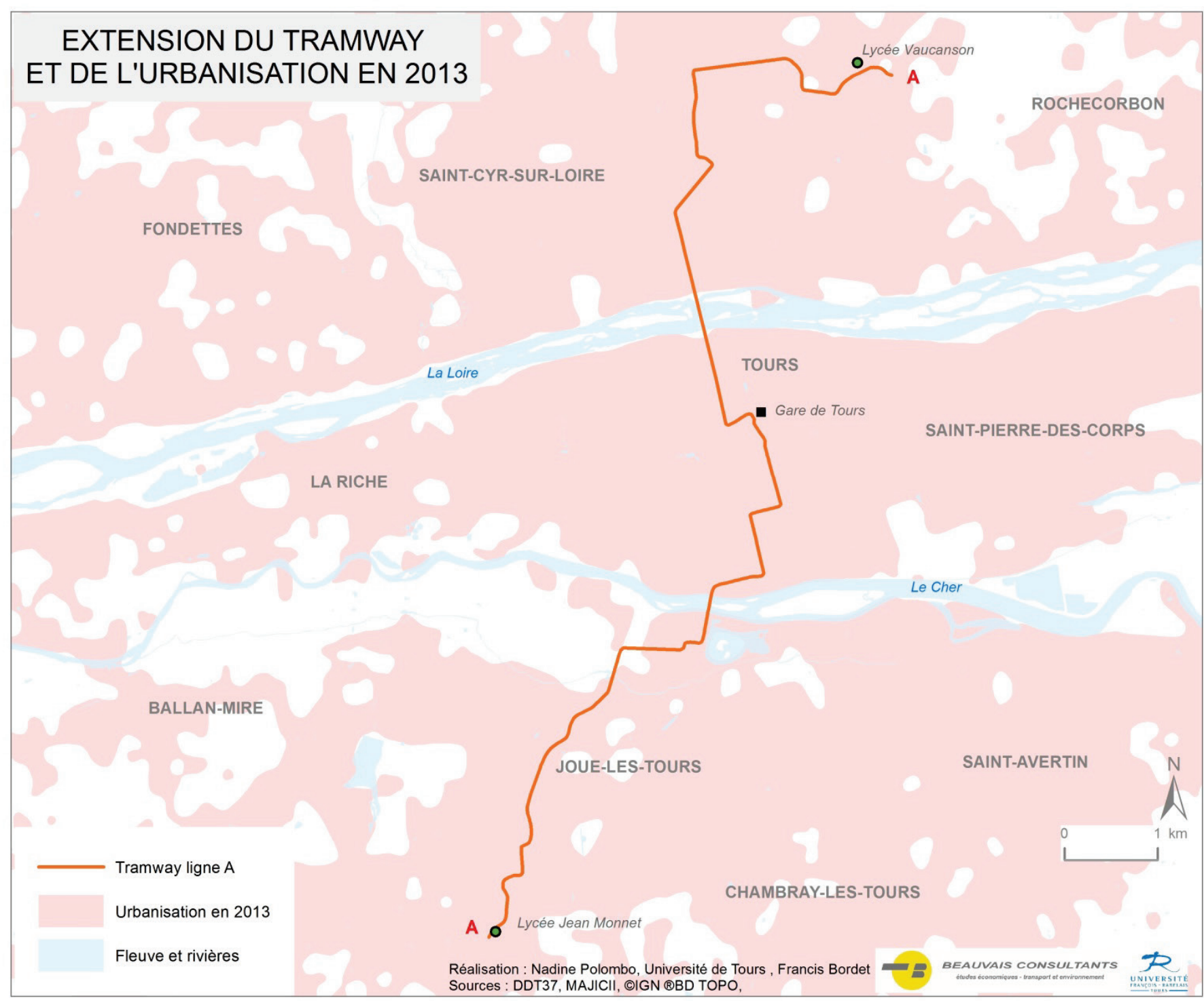

Figure 2 «Extension du tramway et de l'urbanisation en 2013 »

\section{$\underline{2}$ - La technique}

\section{La voie, l'alimentation, les stations}

En 1913, les sites propres n'existaient pas (sauf un petit tronçon à proximité de Luynes) ; le tramway partageait la chaussée avec les voitures à chevaux, les vélos, les piétons et quelques rares automobiles.

Les tramways circulaient sur des voies uniques (sauf sur l'axe rue Nationale / avenue de Grammont, qui est à double voie).

L'écartement était passé du normal au métrique de façon à permettre la liaison avec les chemins de fer départementaux.

La traction vapeur avait disparu en 1912 et toutes les lignes étaient maintenant électrifiées. Le captage de l'énergie se fait à l'aide d'une perche qui relie la motrice à la ligne aérienne. 
Il existait des kiosques où l'on pouvait acheter des billets à Tours, Vouvray et Rochecorbon mais pour les voyageurs montés en cours de route, c'était le receveur à bord du tramway qui assurait la vente des billets en plus du contrôle de tous les voyageurs.

En 2013, la ligne est en site propre de façon à assurer une bonne vitesse commerciale et une bonne régularité. Deux exceptions toutefois : un tronçon avenue de Grammont à l'approche de la place Jean-Jaurès où le tramway partage sa plate-forme avec les autobus et un tronçon avenue Maginot où les voitures empruntent la chaussée du tramway.

La voie est double tout au long du parcours et l'écartement de 1,435 m.

L'alimentation électrique s'effectue sur $88 \%$ du parcours par une ligne aérienne. Elle s'effectue par le sol sur $1.800 \mathrm{~m}$ dans la partie historique c'est-à-dire de la gare à la place Choiseul). La plate-forme est végétalisée sur 6,5 km (sur un total de $15 \mathrm{~km}$ ).

Les stations, au nombre de 29 , sont équipées d'automates de vente des billets. Il n'est pas possible d'acheter son billet à bord.

\section{Le matériel roulant}

En 1913, le parc était constitué de 36 motrices à raison de 20 motrices Thomson affectées aux lignes urbaines et de 16 motrices La Buire affectées aux lignes suburbaines. Les premières étaient généralement équipées de deux moteurs de $25 \mathrm{CV}$ et font 7,60 $\mathrm{m}$ de long et les secondes de deux moteurs de $44 \mathrm{CV}$ et font 8,85 $\mathrm{m}$ de long. Toutes étaient à deux essieux. La capacité était de 36 places dont 20 assises. Aux motrices s'ajoutaient des remorques, fermées ou ouvertes. Les premières étaient souvent d'anciennes motrices transformées en remorques. Les dernières qui étaient dites "baladeuses » offraient 7 bancs de 4 places chacun et 8 à 10 places debout par plate-forme à chaque extrémité. Tours a toujours eu un parc important de baladeuses, très employées les dimanches et jours de fête à la belle saison pour se rendre à Vouvray, à SaintAvertin ou à Luynes.

L'exploitation au quotidien nécessitait 30 motrices et 15 remorques. Le parc des motrices était affecté comme suit : 8 sur la ligne A, 6 sur la B, 4 sur la C, 4 sur la D, 2 sur Vouvray, 2 sur LuynesFondettes, 1 sur la Tranchée, 2 sur St-Avertin / Azay-sur-Cher, 1 sur Pont-Cher. Ce matériel parcourait 1,8 million de kilomètres par an soit à raison d'un parc, réserve comprise, de 36 motrices, une moyenne de $50.000 \mathrm{~km}$.

Ajoutons que les motrices étaient éclairées mais pas chauffées (même l’hiver).

En 2013, le réseau dispose de 21 rames. Chacune se compose de 7 éléments et a une longueur totale de 43,7 m. Elles peuvent emporter 300 voyageurs. Ces rames sont climatisées et peuvent rouler à $50 \mathrm{~km} / \mathrm{h}$. A bord, un conducteur, mais plus de receveur. Les rames assurent 1,3 million de $\mathrm{km}$ par an, soit environ $62.000 \mathrm{~km}$ par an et par rame ce qui est à peine supérieur au chiffre atteint il y a un siècle.

Par ailleurs, les nuisances sonores imputables au tramway ont été drastiquement réduites, sans parler de l'effet absorbant du gazon. 


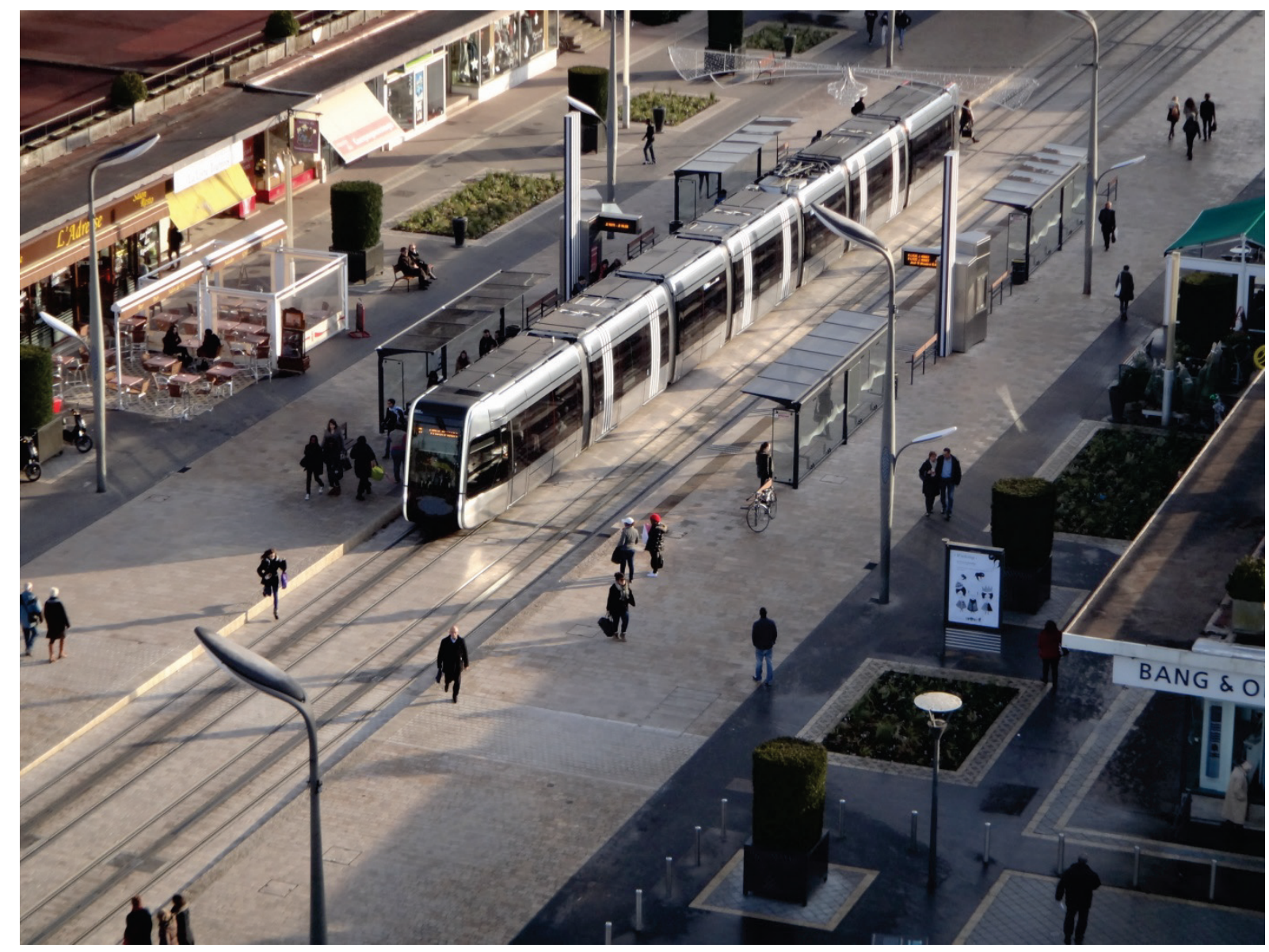

Station de tramway Anatole France (photo J.M. Beauvais)

\section{$\underline{3 \text { - Le service }}$}

\section{L'intensité de l'offre}

En 1913, sur le réseau urbain, le service commençait à $7 \mathrm{~h} 30$ et finissait à $21 \mathrm{~h}$ ou $21 \mathrm{~h} 30$ selon la saison. La fréquence était de 7,5 minutes sur les lignes $A$ et $B$, et de 11 minutes sur les lignes $C$ et D. Sur le réseau suburbain : 6 allers et retours par jour vers Luynes, 6 vers Fondettes, 12 vers Vouvray, 26 vers Saint-Avertin.

Dans le cas de la ligne $\mathrm{A}$, la ligne la plus chargée (près de 2 millions de passagers par an) qui allait de la barrière de Vouvray au carrefour de Verdun $(4,5 \mathrm{~km})$ et qui transportait près de 2 millions de passagers par an, l'amplitude était de 14 heures par jour, la fréquence de 8 par heure, et la capacité d'une rame avec une remorque de 80 places environ. Le nombre de places kilomètres offertes par km de ligne était donc de 112 passages $\mathrm{x} 80$ places $=12.544$ places $\mathrm{x}$ kilomètres.

Aujourd'hui, pour un jour de semaine hors vacances scolaires, le nombre de passages est de 135 par jour. En effet, entre $7 \mathrm{~h}$ et $20 \mathrm{~h}$, il passe une rame toutes les 7 minutes environ mais la fréquence tombe un peu en début de journée et en fin de journée, sachant que le premier départ a lieu à $4 \mathrm{~h} 41$ et la dernière arrivée à $0 \mathrm{~h} 28$. Le nombre de places kilomètres offertes par $\mathrm{km}$ de ligne est donc de 135 passages $\mathrm{x} 300$ places $=40.500$ places $\mathrm{x}$ kilomètres. 
Au niveau quantitatif, l'offre par km de ligne ${ }^{2}$ est donc plus de 3 fois plus importante en 2013 qu'en 1913 : la différence tient essentiellement à l'augmentation de la capacité d'emport des rames.

\section{La durée des parcours}

En 1913, le trajet entre Tours et Vouvray, soit $10,5 \mathrm{~km}$, durait 45 minutes. La vitesse commerciale était donc sur cette ligne suburbaine de $14 \mathrm{~km} / \mathrm{h}$ et on peut penser qu'elle était sensiblement inférieure sur les lignes urbaines en raison du nombre plus important d'arrêts par kilomètre.

Aujourd'hui, le temps de parcours de bout en bout est, selon l'heure, de 47, ou 48 ou 49 minutes ; il est peu sensible aux encombrements. S'il faut 48 minutes pour parcourir $15 \mathrm{~km}$, la vitesse commerciale est donc de $19 \mathrm{~km} / \mathrm{h}$.

La vitesse commerciale s'est donc sensiblement améliorée en 100 ans.

\section{La tarification}

Avant guerre, les billets étaient vendus uniquement à l'unité (il n'y avait pas d'abonnements). En 1910, le tarif urbain était passé de 10 à 15 centimes pour satisfaire une augmentation de salaires. Il n'y avait pas non plus de tarif unique : les tickets valaient entre 15 centimes (cas d'un petit trajet) et 85 centimes (cas de Tours / Azay-sur-Cher). Cette année là, un kg de pain valait 42 centimes et une heure de travail était payée 34,5 centimes (cas d'un manœuvre sans qualification professionnelle). Il fallait donc 49 minutes de travail pour acheter un $\mathrm{kg}$ de pain et entre 26 et 148 minutes de travail pour acheter un billet de tramway.

Au 1 er juillet 2013, le voyage à l'unité est vendu 1,40€ (pour une heure avec possibilité de correspondance). Compte-tenu des nombreuses réductions offertes aux voyageurs occasionnels et des abonnements proposés aux voyageurs fréquents, le voyage moyen revient plutôt à $0,74 €$. Selon l'INSEE, le pain vaut 3,47 € par kg et le SMIC atteint 9,43€ par heure. Il faut donc 22 minutes de travail pour acheter un $\mathrm{kg}$ de pain, 9 minutes de travail pour pouvoir faire un voyage en tram.

Le voyage en tramway coûtait donc au moins trois fois plus cher il y a un siècle, compte tenu du pouvoir d'achat de l'époque. Cela devrait se retrouver dans la structure de la clientèle et son volume.

Rappelons qu'à l'époque, la compagnie versait une redevance à la ville alors qu'aujourd'hui, c'est la communauté d'agglomération et les entreprises qui financent la différence entre les produits et les charges de l'exploitant des transports urbains, Fil Bleu.

\section{4- La fréquentation}

\section{Nombre de voyages par an}

\footnotetext{
${ }^{2}$ La comparaison porte sur deux lignes (la principale en 1913 et la seule en 2013) et non pas sur l'ensemble du réseau : aujourd'hui les parcours en tram représentent 1,3 million de km et ceux en autobus, inexistants à l'époque, représentent 9,0 millions de km.
} 
En 1913, le réseau transportait environ 6 millions de voyageurs dont les trois quarts sur les 4 lignes urbaines. La ligne la plus fréquentée, la ligne $\mathrm{A}$, comptant à elle seule pour 2 millions de voyages.

Les lignes suburbaines étaient moins fréquentées mais voyaient leur activité renforcée le dimanche à la belle saison. Les Tourangeaux allaient prendre l'air à la campagne ou danser dans les guinguettes comme à Vouvray. La principale ligne suburbaine, celle de Vouvray, ne voyait pas plus de 600.000 voyageurs par an.

Aujourd'hui, le réseau (autobus compris) assure 24 millions de voyages par an, soit 4 fois plus. Sur ce total, la ligne de tramway compte pour $40 \%$ environ c'est-à-dire qu'à elle seule elle transporte plus de passagers que tout le réseau en 1913. Un jour hors vacances scolaires, elle transporte près de 55.000 voyageurs.

\section{Typologie de la clientèle}

Aujourd'hui, les femmes représentent $62 \%$ de la clientèle de Fil Bleu. Les étudiants/lycéens/collégiens/écoliers comptent pour $52 \%$ de la clientèle de Fil Bleu.

Mais qu'en était-il à la veille de la première guerre mondiale ? Il est très difficile de répondre à cette question. On peut néanmoins avancer qu'une partie de la clientèle était féminine mais qu'elle était très probablement minoritaire. De même que les scolaires et les étudiants étaient encore rares.

A la lecture des photos et des cartes-postales de l'époque, il semble que la clientèle soit presque toujours endimanchée. Effectivement, on s'habillait, on se coiffait pour sortir. Et notamment à Tours qui était une ville bourgeoise. Mais l'échantillon peut être biaisé : photos prises lors d'un jour férié, voire à l'occasion d'une inauguration. Quant aux lycéens, ils étaient encore peu nombreux dans les transports publics car une très faible partie de la population poursuivait des études. Et puis, le prix du billet, on l'a vu, n'était pas si abordable ; les abonnements n'existaient pas encore.

Finalement, en un siècle, la clientèle s'est démocratisée. C'est vrai de l'automobile mais c'est aussi vrai du transport collectif. 


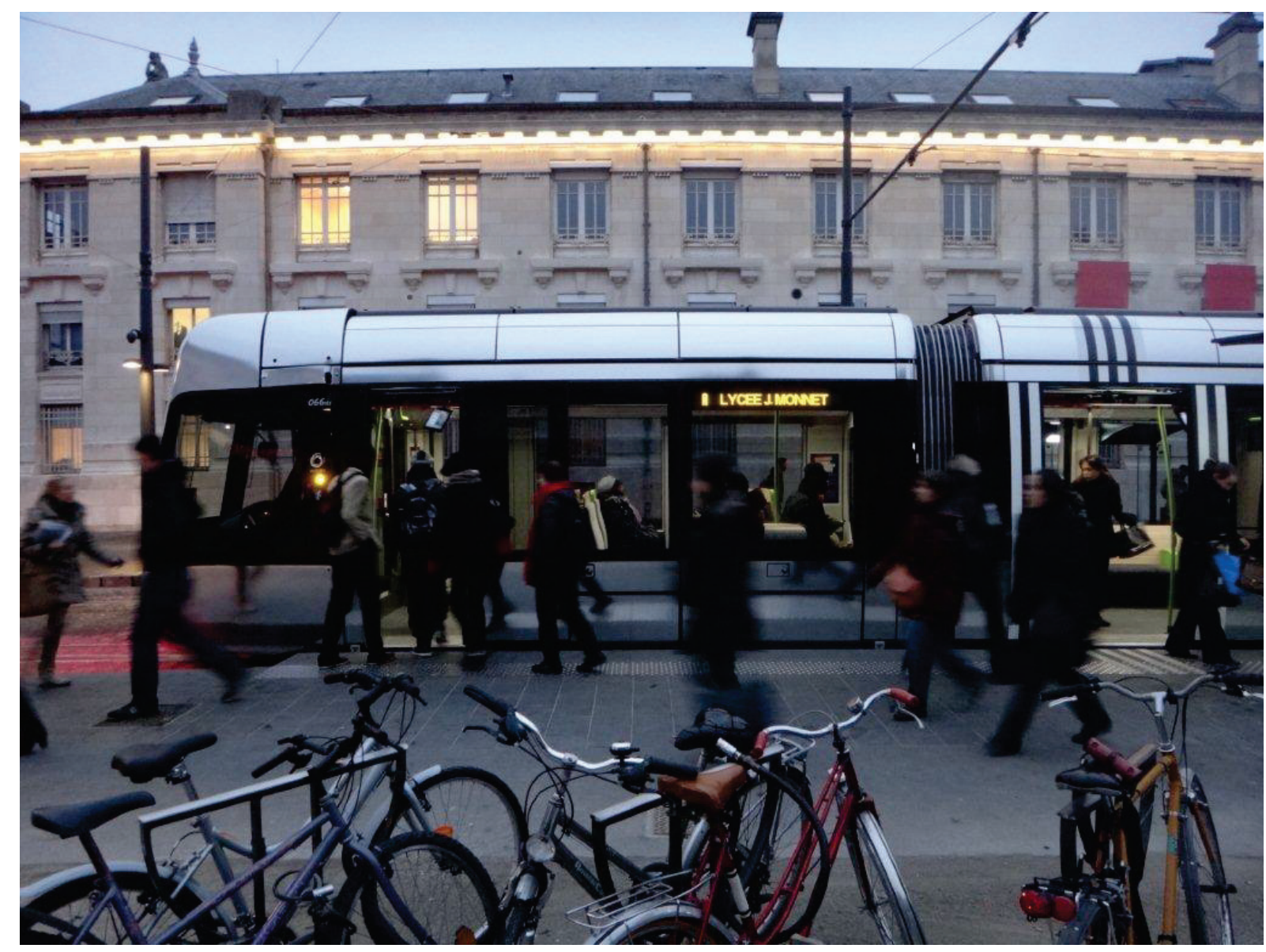

Gare de Tours au petit matin : clientèle jeune pour le tramway (Photo J.M. Beauvais)

\section{$\underline{5 \text { - Le design }}$}

Ici le design est entendu au sens large c'est-à-dire qu'il n'est pas limité aux préoccupations esthétiques mais se situe au niveau de la conception du système dans son ensemble.

\section{La conception}

En 1877, année de la mise en service de la première ligne (à traction hippomobile) de tram entre la barrière de Grammont (carrefour de Verdun) et la barrière de Vouvray (quai Paul-Bert), il s'agissait avant tout d'une opération financière. La compagnie qui reçoit la concession apporte le capital nécessaire pour l'investissement, encaisse les recettes et ristourne un pourcentage des bénéfices à la ville. Les compagnies rivalisent entre elles (Compagnie générale française de tramways, Société des tramways à vapeur, Compagnies des tramways de Tours) jusqu'à ce que la guerre de 1914-1918 marque l'arrêt des concessions.

Aujourd'hui, la Collectivité s'implique plus, notamment financièrement, et, plus généralement, la coordination entre les acteurs l'emporte maintenant sur la concurrence.

Le tramway de Tours, qui est un tramway "à la française », est le fruit d'une remarquable union entre l'excellence des métiers créatifs et techniques, le savoir-faire des industriels et des opérateurs de transport public. Le design est intégré dès le stade de l'idée et intervient à tous les niveaux de développement pour répondre aux attentes en termes d'usage, de fonctionnalité, 
d'ergonomie, de sécurité, d'accessibilité, de respect de l'environnement, d'esthétique et de rentabilité.

C'est non seulement un moyen de transport mais aussi un outil d'urbanisme, de reconquête de l'espace public, de lien social. Là où le tram passe, la ville repousse ! Il accentue le sentiment d'appartenance. Il véhicule l'identité de l'agglomération tourangelle au même titre que la Loire, les jardins, le patrimoine bâti et Rabelais, Ronsard, Balzac, Léonard de Vinci. D'ailleurs des citations de ces auteurs figurent sur les stations du tram.

Enfin, le principe d'une intervention de façade à façade a favorisé la mutation de l'image de la ville.

"Changer de point de vue sur le projet et le penser comme un territoire nouveau de la ville, non plus sur $15 \mathrm{~km}$ de long mais sur $15 \mathrm{~km}^{2}$ intégrant ses zones d'influence de part et d'autre de la ligne. Et sur ce territoire nouveau, donner le meilleur de l'expression urbaine, faire de la ligne une seule et même ouvre urbaine inédite, à habiter, totalement accessible par tous et en dialogue permanent avec la ville. Lui donner la meilleure part de son identité en faisant parler la culture et l'art». Régine Charvet-Pello (Manifeste d'ensemble(s), la ligne pour le tramway de Tours, RCP, août 2013

\section{L'esthétique proprement dite}

Au début du 19 ème siècle, sur près de $5 \mathrm{~km}$, l'alimentation se faisait à l'aide de plots dits Diatto (du nom de l'inventeur, un ingénieur de Turin) situé au milieu de la voie. Il s'agissait d'éviter les nuisances visuelles liées aux fils à l'approche des lieux remarquables, comme la cathédrale StGatien, la gare de Tours, la place Anatole-France ou bien le grand théâtre. Ce système deux fois plus coûteux en investissement que le trolley, s'est révélé catastrophique en coûts de fonctionnement et en termes de sécurité (cas de chaussures avec des fers ou des clous des chevaux). Si bien que ce système avait quasiment cessé de servir en 1913. Mais cela montre que les préoccupations esthétiques qui ont conduit le maire, Jean Germain, à attendre que le système d'alimentation par le sol expérimenté à Bordeaux soit complètement au point, ne datent pas d'aujourd'hui.

Aujourd'hui le souci de beauté ne se limite pas à la question de l'alimentation. On citera, par exemple, la rame elle même avec ses allures de fermeture éclair ou de smart phone géant, sa livrée qui reflète la couleur sable des bâtiments en tuffeau, son aménagement intérieur avec les bourgeons qui ponctuent le creux de barres de préhension et qui ont été réalisés par les élèves des lycées techniques de l'agglomération tourangelle, le pont sur le Cher qui s'illumine lorsqu'il fait nuit au passage du tram, ...

Bien sûr, le beau peut aussi être très fonctionnel. C'est le cas des raies de Daniel Buren. Elles figurent sur les quatre faces du totem qui permet de repérer les stations de loin. Elles sont aussi dessinées au sol à l'endroit où les portes de la rame vont s'ouvrir (si le conducteur est habile), ce qui permet de savoir où se placer pour ne pas gêner la descente des voyageurs.

Finalement, l'importance accordée au design constitue une différence par rapport au siècle dernier et aussi une spécificité par rapport aux autres réseaux qui n'ont pas été aussi loin que Tours dans cette direction. 


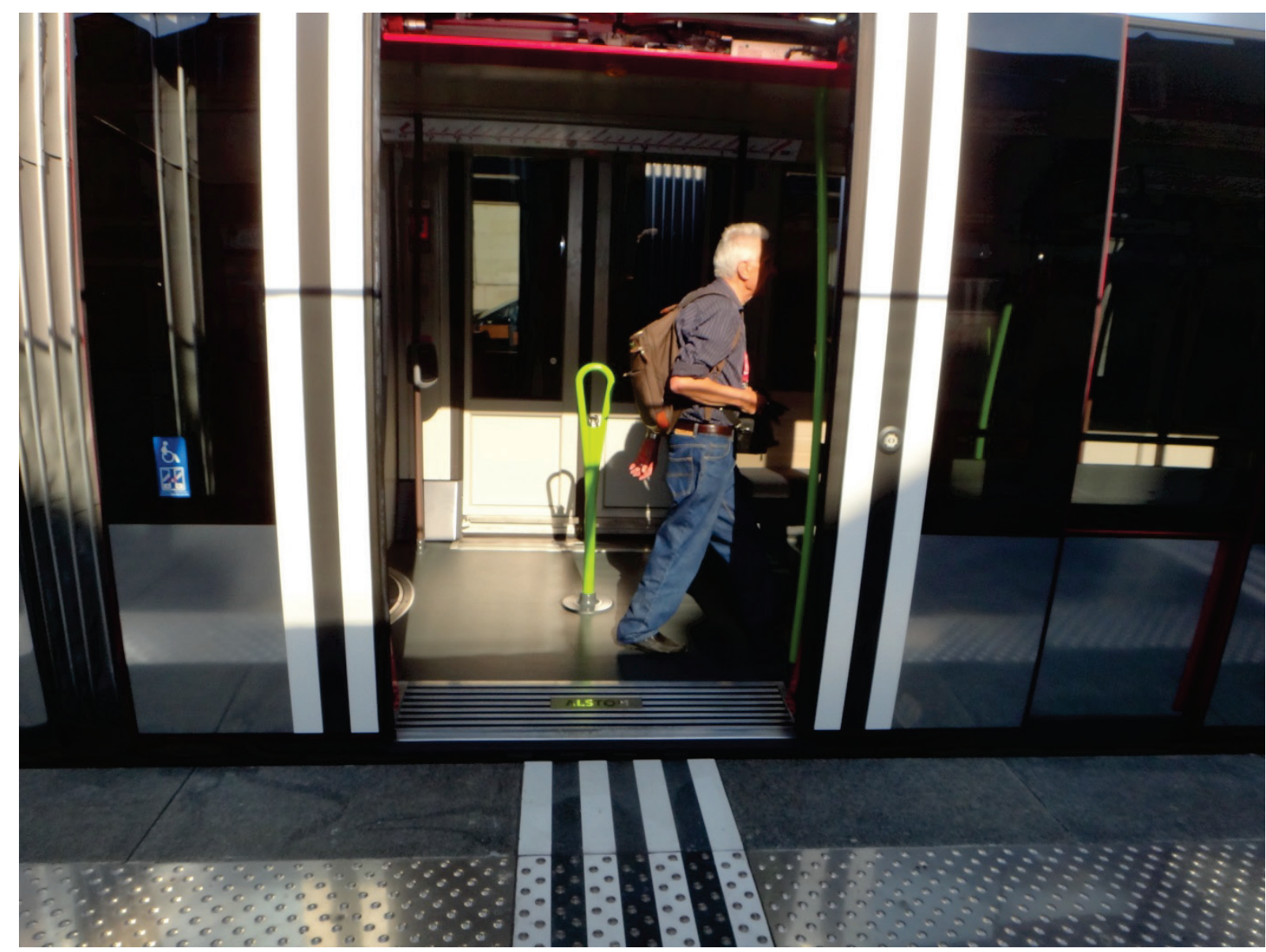

Les bandes Buren en continuité sur la carrosserie et le quai (Photo J.M. Beauvais)

\section{Conclusion}

Le tramway d'aujourd'hui n'est pas comparable à celui d'il y a un siècle en ce sens que l'offre est plus intense grâce à la grande capacité des rames, la vitesse commerciale supérieure grâce au site propre, les prix plus abordables grâce aux abonnements, sans parler de l'amélioration du confort (moins de bruit, une température agréable, des sièges plus confortables, ...).

Ce qui démarque le nouveau tram de Tours tant par rapport à l'ancien tram que par rapport aux tramways de beaucoup d'autres villes de par le monde, c'est la place centrale accordée au design. Un design qui a permis au tram d'être accepté dans cette ville sans véritable culture industrielle.

À ce jour, mais cela peut changer, le point faible reste que le tram de Tours demeure limité à une seule ligne. À la veille de la première guerre mondiale, le réseau était 3,6 fois plus étendu alors que la population était 2,6 fois moindre. Une ligne B et une ligne $C$ voire une ligne D pourraient être tracées vers St-Pierre-des Corps, La Riche, St-Avertin, Chambray, Rochecorbon, St-Cyr-surLoire et Fondettes.

Pourquoi ces communes devraient-elles renoncer aux avantages apportés par le tramway: capacité de transport, absence de place de stationnement à rechercher, temps de parcours garanti, moins d'accidents de circulation, moins de pollution atmosphérique, moins de bruit, moins de gaz à effet de serre ? Souvenons-nous des années 1990, quand la rue Nationale était encombrée par les voitures et que les engins de secours ne pouvaient pas avancer. 


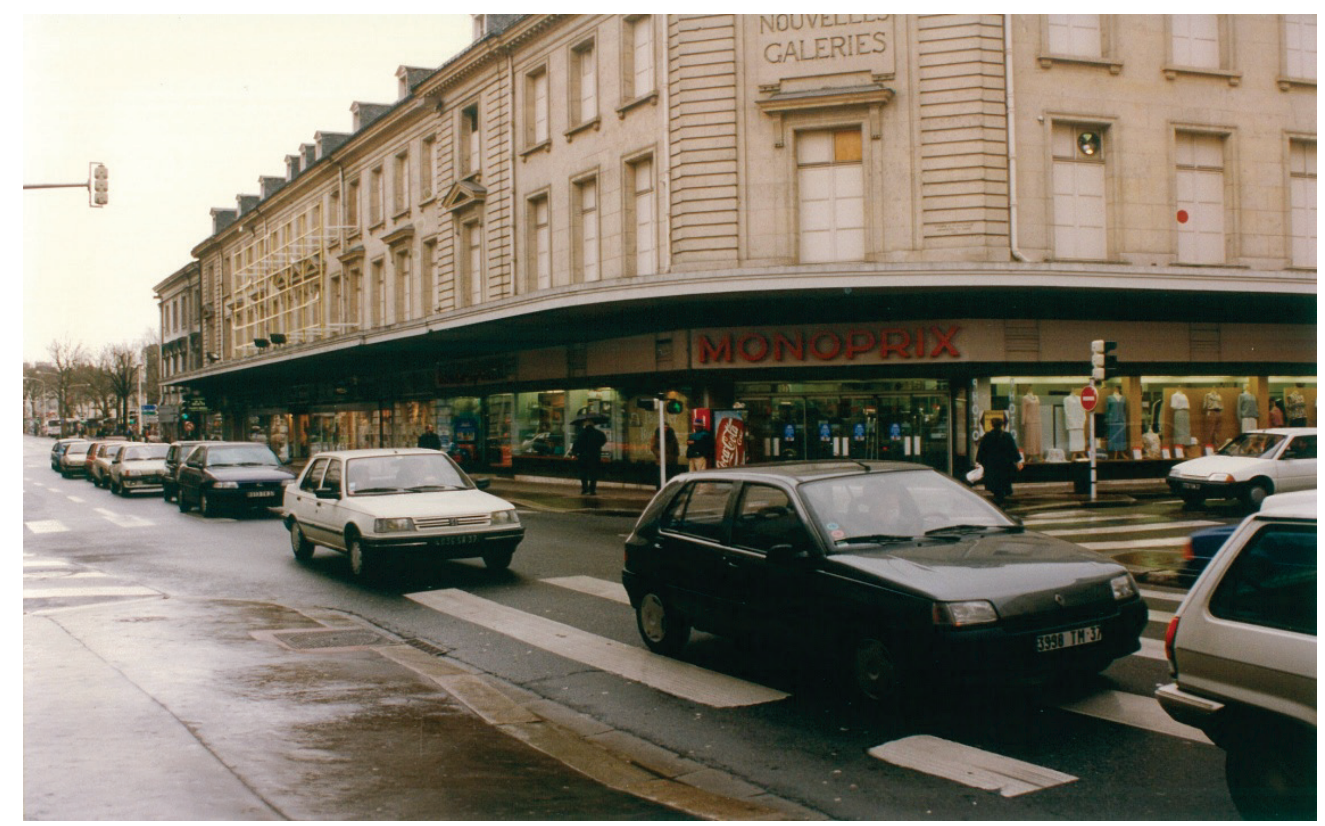

La rue Nationale à Tours en 1997 (photo Jean-Jacques Moreau).

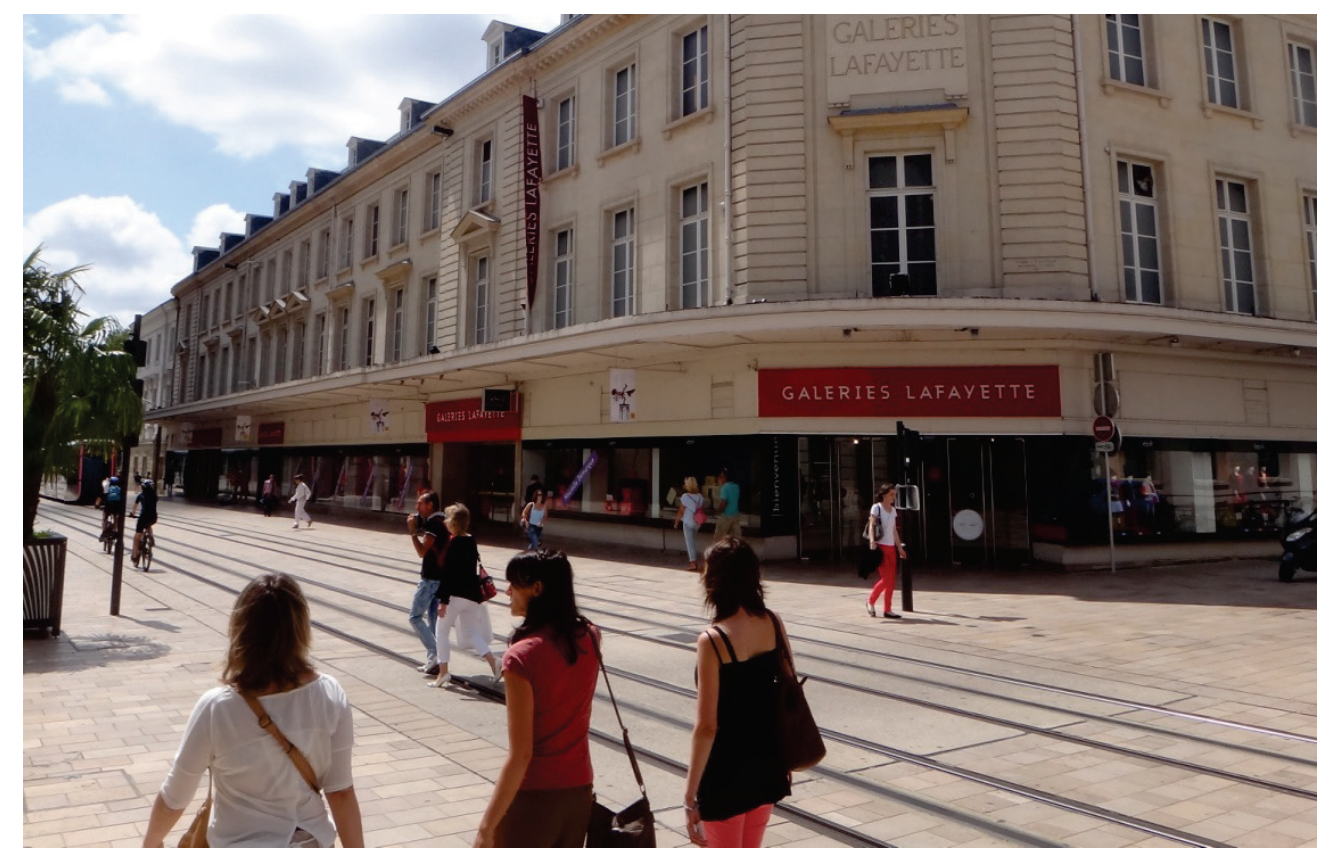

La rue Nationale à Tours en 2014 (photo Jean-Marie Beauvais).

Au début du XXème, siècle le tram apportait à Tours une forme de révolution dans le transport urbain ; en 2013, après un hiatus de 64 ans, il a de nouveau transformé la ville. Pourquoi ne pas continuer dans cette voie du renouveau?

$27 / 07 / 2014$ 